\title{
Soft Textile Electrodes for EEG Monitoring
}

\author{
Johan Löfhede, Fernando Seoane and Magnus Thordstein
}

\begin{abstract}
There is a need for long term monitoring of the brain during intensive care. This is e.g. the case for newborn babies that have been exposed to hypoxia during delivery. Electroencephalography (EEG) is the technique of choice. To get a clear and detailed view of the brain activity a large number of EEG electrodes should be used. Applying traditional electrodes one by one is a time-consuming and technically demanding work and therefore electrode caps are sometimes used. The existing caps have however been found to be suboptimal for long term monitoring because they may induce too high a pressure on the scalp of the babies. We have tested three different types of textile electrodes with regard to their potential use for EEG monitoring. The results show that soft conducting textile materials can indeed be used for EEG monitoring.
\end{abstract}

\section{INTRODUCTION}

$\mathrm{W}$ HEN an individual is in need of intensive care, cardiovascular and respiratory parameters are routinely monitored. The function of the organ most vital to the eventual recovery of the individual, the brain, is however often neglected. This goes also for neonatal intensive care. When a baby is being born there is a risk for complications that reduce the blood gas exchange to the fetus during delivery. This can lead to hypoxia, acidemia and potentially cause brain damage. When complications are expected or suddenly take place, the baby is monitored closely during the delivery and is then, if necessary, transferred to a neonatal intensive care unit (NICU). It is however difficult to determine at an early stage if the baby's brain is recovering, if brain damage is still a threat or if it already has occurred [1]. At the NICU parameters such as heart rate, blood pressure, oxygen saturation and other measures are monitored regularly [2], but these parameters only assess the general condition of the baby. To monitor the function of the brain itself, a more direct way is to measure the electroencephalogram (EEG); a measure of the electrical signals produced by the brain. This makes possible continuous monitoring of the brain over long time with high time resolution. Recently developed automatic classification methods can be used to simplify the interpretation of the complex EEG signal $[3,4]$.

Manuscript received July 9,2010 . This work was supported in part by the Swedish state under the ALF-agreement and by the Margarethahemmet foundation.

J. Löfhede is with the School of Engineering, University of Borås, Borås, Sweden. (phone: +46 7398496 69; fax +46 3343540 08; e-mail: johan.lofhede@hb.se.)

F. Seoane is also with the School of Engineering, University of Borås.

M. Thordstein is with the Institute of Neuroscience and Physiology, Section for Neuroscience and Rehabilitation, Sahlgrenska University Hospital, Göteborg, Sweden.

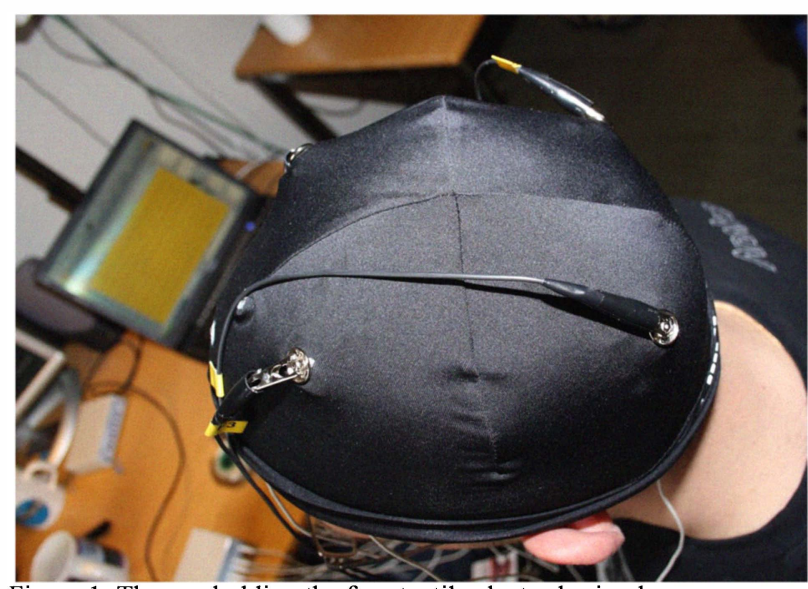

Figure 1: The cap holding the four textile electrodes in place.

Traditional EEG monitoring systems (e.g. [5]) usually relies on a limited number of channels. However, important pathological brain activity, e.g. seizures, can be very localized and using a too sparse electrode grid may allow these events to be missed by the classification system. Using traditional electrodes that are placed one by one on the scalp and attached by gel is a time consuming and technically demanding work. Instead, electrode caps can be used. There is a very limited selection of electrode caps for this patient group, utilizing plastic cups containing the gel and the actual electrode [6]. When doing some limited testing of these we have found that it under some conditions makes marks on the scalp of the babies. This indicates that the pressure of the cup onto the scalp may cause problems when used during long-time monitoring.

To avoid the problem with pressure points in the electrode cap we propose a solution where the cup and electrode are replaced by a patch of soft conductive textile. Textile electrodes (or "textrodes") have previously been used for $e$.g. ECG monitoring [7], where the signals are in the range of millivolts rather than microvolts as in the EEG case. In the following we will show results that demonstrate that textile electrodes can produce an EEG signal that is comparable with the one acquired using conventional high-quality electrodes.

\section{METHODS}

A tight-fitting thin cap was prepared with small holes in the approximate locations of the electrodes P3, P4, F3, F4 according to the international 10-20 system (Figure 1). The textile electrodes were tested using either physiological saline solution or standard electrode gel (Nihon Kohden Elefix). They were fastened to the cap using snap-buttons, 
and cables with crocodile clips were connected to the outside part of the snap button. Standard high-quality sintered eight mm EEG electrodes (In Vivo Metric E272-HS, Figure 2) were placed within five $\mathrm{mm}$ of the textile electrodes using the same standard electrode gel. Standard electrodes were also used for reference and ground and placed on the midline of the forehead.

The signals were digitized at a sampling frequency of $1000 \mathrm{~Hz}$ using a DC-coupled EEG amplifier (NeuroS4U, Medical Computer Systems, Moscow, Russia) and a laptop running the SACS signal acquisition and archiving system [8]. After recording, the signals were exported to Matlab where DC offset was removed by subtracting a fitted straight line from the data (detrending). For the time-domain plots, power line interference was reduced using a notch filter at $50 \mathrm{~Hz}$ and the signals were band-pass filtered from 0.5 to 70 $\mathrm{Hz}$.

The signals from the standard and textile electrodes were compared using visual inspection of the data in the time and frequency domains. The power spectra $(0-45 \mathrm{~Hz})$ were estimated using the Welch method (using eight modified periodograms with a Hamming window and $50 \%$ overlap [9]) and based on $10 \mathrm{~s}$ epochs of data. No filtering except detrending was applied on the data before spectrum estimation.

For this paper, plots of signal in the time- and frequency domains have been selected from signal segments where one subject is awake, sitting comfortably with his eyes closed. These circumstances induce the alpha rhythm $(7.5-13 \mathrm{~Hz})$ that is well-known and readily identified over the posterior parts of the head.

The three types of textile electrodes (Figure 2) were made from the following materials:

\section{A. Electrode type I}

The material used for the first electrode type was a

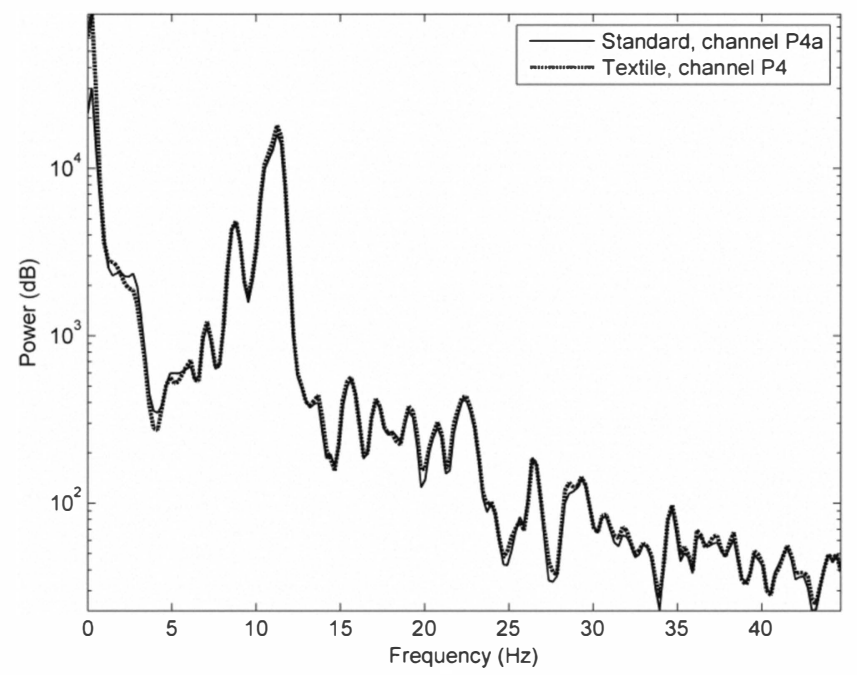

Figure 3: Frequency spectra for the signal in figure 5 (electrode type II + gel)

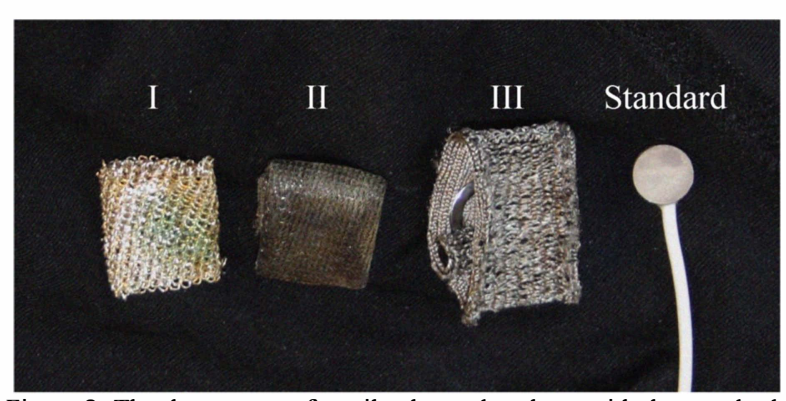

Figure 2: The three types of textile electrodes along with the standard electrode.

metallic silver thread that was knitted in a circular knitting machine into a metallic mesh fabric at the Swedish School of Textiles in Borås.

\section{B. Electrode type II}

The material for the second electrode type was woven from a nylon substrate coated by pure silver (Silver mesh fabric, Less EMF inc., Albany, NY, USA).

\section{Electrode type III}

The material for the third electrode type was made by $15 \%$ nylon, $30 \%$ conductive fibers, $20 \%$ Spandex and $35 \%$ polypropylene. The material was knitted, and is similar to terrycloth (Textronics inc., Wilmington, Delaware, USA).

\section{RESULTS}

According to our measurements, electrode type I produces a rather poor signal quality, both when using saline solution and when using gel. Electrode type II gives good signal quality when using gel (figure 3 and 5), almost indistinguishable from the signals acquired using the standard electrodes, but did not work very well when wetted with saline. Electrode type III on the other hand gives as good results when the electrodes are wetted with a saline solution (figure 4 and 6 ) as when they are used with gel.

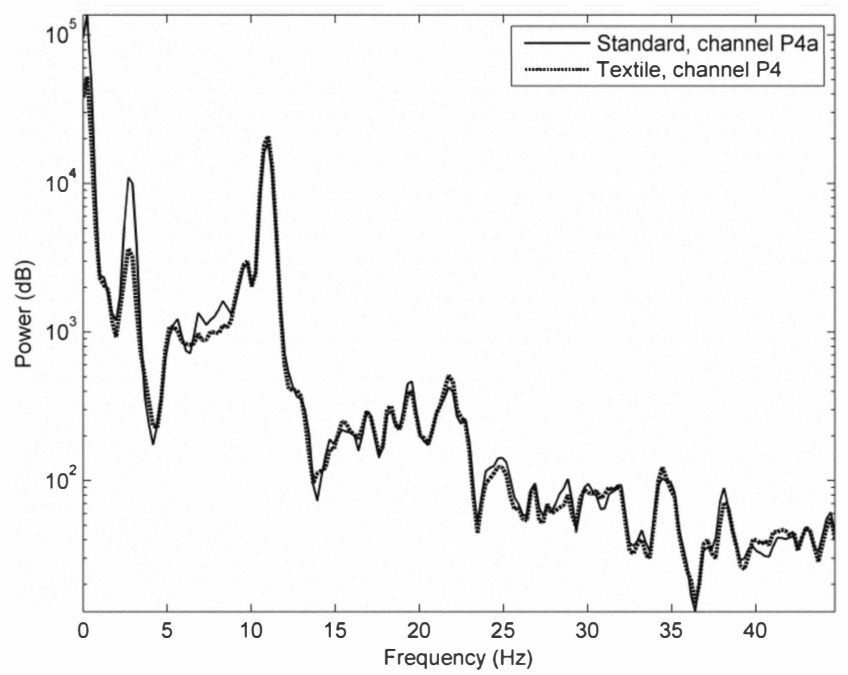

Figure 4: Frequency spectra for the signal in figure 6 (type III + saline). 


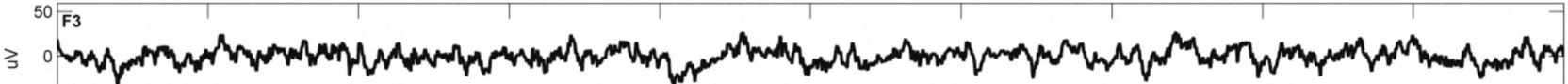
$50-50$

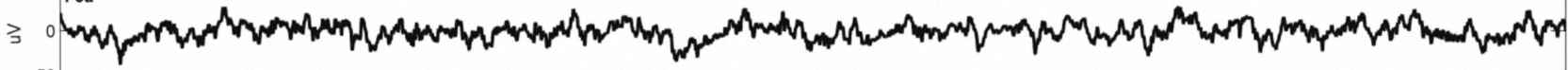
${ }_{50}^{-50} \overline{\mathrm{F}_{4}}$

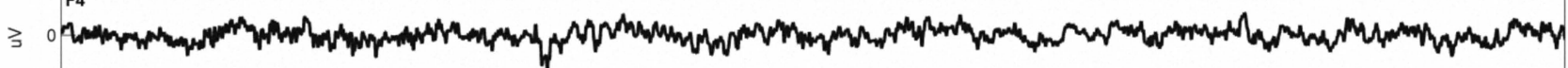
$-50-$

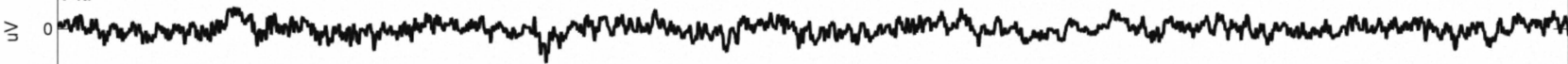
$50-$

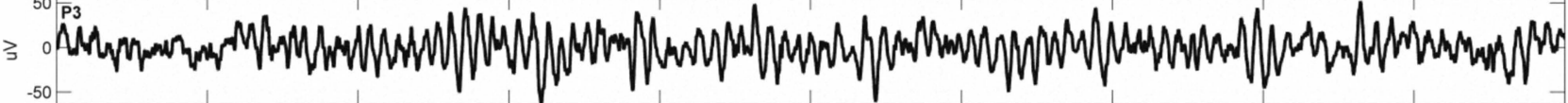
${ }^{50}{ }^{-50}{ }^{\mathrm{P}} 3 \mathrm{a}$

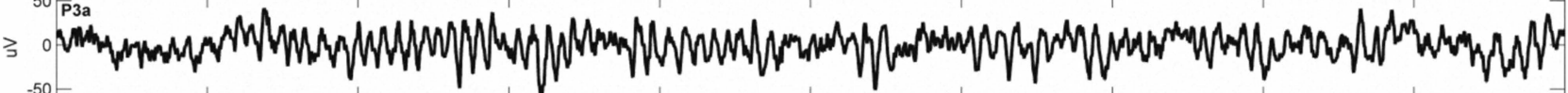
$-50-$

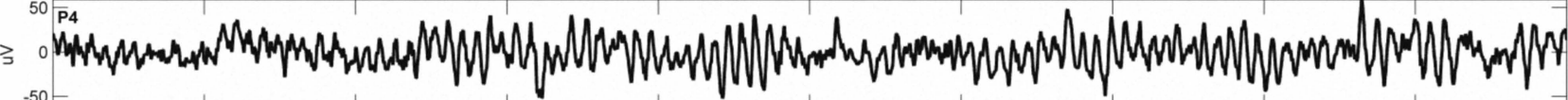
$-50-$ 30 . 30 .

$16: 00: 35$

Figure 5: Ten seconds of EEG signals captured from an adult subject with closed eyes. The channels marked with an "a" are standard electrodes, the others are electrode type II with gel.

30 =30

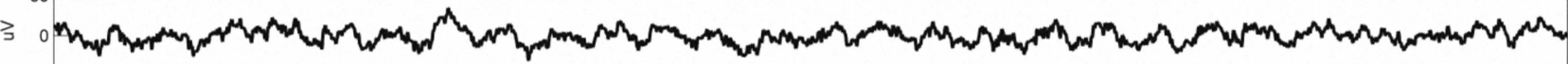
${ }_{50}^{-50} \mathrm{F4}$

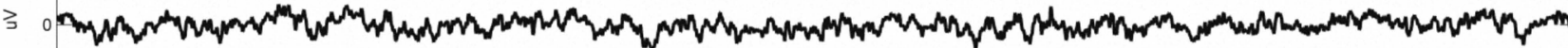
$-50-$

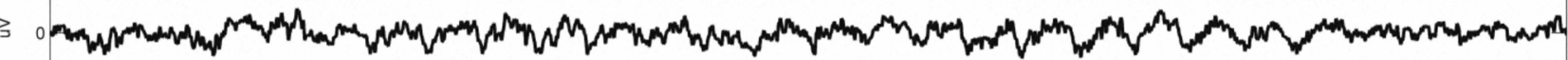
$-50$

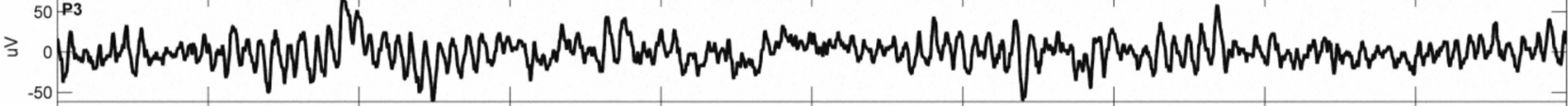
-50
50 P3

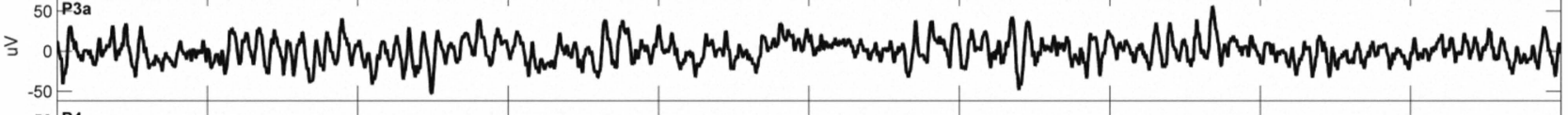
30.

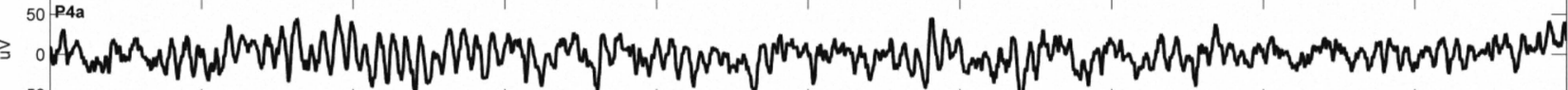
16:13:48

Figure 6: Same subject as in figure 5, but this time with electrode type III wetted with a saline solution. 


\section{DISCUSSION}

The textile electrodes tested in this work ranges from knitted metallic silver yarn to a soft terrycloth containing conducting fibers. Since measuring EEG is a passive process where the electric fields produced spontaneously by the brain are measured, it is hard to make any absolute comparisons; each time a signal is measured it is different, depending on a number of factors including the state of the test subject. We have made simple textile electrodes, placed them close to standard state of the art sintered electrodes, compared the signals visually in both time and frequency domain and have hopefully been able to make a fair comparison.

The metallic silver (electrode type I) produced the worst signal quality. This may in part be due to the physical properties of the electrodes, they creased easily in a way that may have given them a less beneficial contact with the skin. Electrode type II also creased slightly, but produced rather good signals when used with electrode gel. A possible explanation for the difference might be that this material was made by thinner yarn, giving them a larger total surface area. The terrycloth material on the other hand works well both with gel and with saline solution. This may be due to the fact that this type of material is very good at absorbing liquids, and that it therefore can contain a lot of saline solution while the more metallic textiles loose moisture more quickly. The material also has a very large surface area due to the way it is knitted. A final advantage of this material is that it is very soft, much like the material commonly used in towels. This property may be very useful in an application intended for long term monitoring, especially in neonates.

\section{CONCLUSION}

We have shown that soft conductive textile materials can indeed be used as EEG electrodes. This may be a very useful fact when designing electrode caps for long-term monitoring, especially of newborn babies.

\section{ACKNOWLEDGMENT}

The authors thank Anna-Karin Proohf and Tommy Martinsson at the Swedish School of Textiles who prepared the textile electrodes.

\section{REFERENCES}

[1] M. Scher, "Perinatal asphyxia: timing and mechanisms of injury in neonatal encephalopathy," Current neurology and neuroscience reports, vol. 1, pp. 175-184, 2001.

[2] S. Nicklin, Y. A. Wickramasinghe, and A. S. Spencer, "Neonatal intensive care monitoring," Current Paediatrics, vol. 14, pp. 1-7, 2004.

[3] J. Löfhede, N. Löfgren, M. Thordstein, A. Flisberg, I. Kjellmer, and K. Lindecrantz, "Classification of burst and suppression in the neonatal electroencephalogram," Journal of Neural Engineering, vol. 5, pp. 402-410, 2008.

[4] J. Löfhede, M. Thordstein, N. Löfgren, A. Flisberg, M. Rosa-Zurera, I. Kjellmer, and K. Lindecrantz, "Automatic classification of background EEG activity in healthy and sick neonates," Journal of Neural Engineering, vol. 7, 2010.

[5] P. F. Prior and D. E. Maynard, Monitoring Cerebral Function Amsterdam: Elsevier, 1986.

[6] S. Vanhatalo, M. Metsäranta, and S. Andersson, "High-fidelity recording of brain activity in the extremely preterm babies: Feasibility study in the incubator," Clinical Neurophysiology, vol. 119, pp. 439445, 2008

[7] M. Catrysse, R. Puers, C. Hertleer, L. Van Langenhove, H. van Egmond, and D. Matthys, "Fabric sensors for the measurement of physiological parameters," in TRANSDUCERS, Solid-State Sensors, Actuators and Microsystems, 12th International Conference on, 2003, 2003, pp. 1758-1761 vol.2.

[8] K. Lindecrantz, R. Bågenholm, F. Göthe, A. Hedström, N. Löfgren, S Nivall, and J. Ouchterlony, "A general system used in monitoring of cerebral and circulatory function in neonatal intensive care," Med Biol Eng Comput, vol. 37, pp. 888-9, 1999.

[9] M. H. Hayes, Statistical Digital Signal Processing and Modeling. New York: John Wiley \& Sons, Inc., 1996. 